\title{
Rest-to-Rest Motion for Planar Multi-Link Flexible Manipulator Through Backward Recursion
}

\author{
G. Le Vey \\ Institut de Recherche en Cybernétique de \\ Nantes, \\ UMR 6597-CNRS 1 rue de la Noë, \\ BP 92101, 44321 Nantes Cedex 3, France \\ e-mail: levey@emn.fr \\ L. Lanari \\ e-mail: lanari@dis.uniromal1.it \\ A. De Luca \\ e-mail:deluca@dis.uniroma1.if \\ Universit degli studi di Roma "La Sapienza," \\ Via Eudossiana 18, 00184 Roma, Italy
}

In this work is considered the problem of rest-to-rest motion in a desired pre-fixed time for planar flexible manipulators. We introduce a simple idea permitting the minimization of end-effector residual vibration when reaching a desired angular equilibrium position, in a pre-fixed desired travelling time. The results hold without considering internal elastic damping effect, using a classical controller with feedforward plus joint feedback terms. The new approach concerns the computation of the feedforward control, which is based on backward integration of the elastic dynamics, starting from a rest position of the flexible arms. This backward integration yields basically elastic trajectories permitting to reach the final desired end-effector position without oscillation. The feedback controller is then used to stabilize locally the actual states along these desired trajectories. However, for fast rest to rest motion, the feedback compensator fails to drive the system states along the desired trajectories, this being due to the relatively large initial elastic error. To overcome this limitation, proper joint motion is planned between the desired initial and final positions through optimization techniques, the goal being the minimization of the initial elastic error associated to these joint trajectories. The optimal planning technique is formulated as a Pontryagin optimal control problem. This scheme is validated via numerical tests as well as experiments on a flexible two-link planar manipulator.

[DOI: $10.1115 / 1.1649976]$

\section{Introduction}

For flexible multi-link manipulators, three interesting control problems hold. The first one concerns the point to point motion in a pre-fixed time, where the final joint position should be reached in a desired finite time both with cancellation of residual tip oscillations (this differs from the regulation problem, where the time motion is not constrained). The second one concerns the tracking of a desired angular trajectory, together with the constraints of eliminating or at least minimizing the residual elastic deflection of the end-effector. The last problem is more challenging and concerns the tracking of desired end-effector operational trajectories; in this case, the end-effector to joint torque model owns a nonminimum phase property, that is well-known to make inversion model a difficult problem.

In this paper, we concentrate on the first problem and propose a solution based on joint trajectory control, avoiding this way the non-minimum phase problems appearing when dealing with endeffector output to joint torque plant controllers.

Many strategies exist for the position control of such systems. Firstly, the regulation problem has been treated by number of authors whom have used simplified linear models, for a one-link flexible arm. For example, in [1], a method based on control input shaping is presented on spring-mass oscillating systems. The method called "ramped sinusoids forcing function" amounts to a decomposition of the input signal in sine, cosine and ramp functions bases. The coordinates of the decomposition are then computed via minimization of the controller energy during the motion and the elastic acceleration at the end of the desired motion. In [2], the oscillating system of spring-mass is also considered to present an optimal shaped trajectory approach. The desired trajectories are shaped such that minimal elastic velocity, acceleration and jerk hold. A novel regulation strategy has been recently intro-

Contributed by the Dynamic Systems, Measurement, and Control Division of THE AMERICAN SOCIETY OF MECHANICAL ENGINEERS for publication in the ASME JOURNAL OF DYNAMIC SYSTEMS, MEASUREMENT, AND CONTROL. Manuscript received by the ASME Dynamic Systems, and Control Division July 28, 2003. Associate Editor: M. Goldfarb. duced in [3]. The scheme is based on wave propagation and absorption techniques, and has been successfully applied to a lumped-parameter mass-spring model. We also cite [4], where it is shown that an open-loop strategy based on shaped-input filters is a simple method to reduce the end-effector vibrations and ensure good performance even when the actual system frequencies are imprecisely known. We refer also to [5] for a linear model based design of input shaper controllers robust with respect to the system natural frequencies and damping coefficients variations. In [6], the authors treat the regulation problem, for the class of multilink manipulator with one flexible link, using dominant elastic vibration frequencies filters added to simple rigid controllers. For the same manipulator class, we refer also to $[7,8]$ for a vibration suppression scheme, based on a closed-loop input shaping technique. The rest to rest problem in fixed desired time is considered in [9], where a full state based controller is used. The dynamics of the system are linearized around an equilibrium point and a full state regulator is then computed. Good results are obtained on a two-link manipulator with flexible forearm. However, a full state feedback needs measurement or estimation of the elastic states, which is not straightforward for practical implementation (see also $[10,11]$ for elastic measurement feedback based schemes). In [12], two algorithms yielding optimal joint trajectories are presented. The obtained rigid trajectories lead to small final elastic residual vibrations. In [13], a method based on the travelling time tuning is presented. Basically, the motion time is augmented until the final deflection remains under a given threshold. A nearby time optimal bang-bang control has been presented in [14]. The control signal is based on a modification of the bang-bang form, where the abrupt variations have been replaced by linear functions. Experimental results on a two-link flexible arm testbed are reported in [15], where the rest to rest motion problem is treated. The desired joint point to point motions are firstly shaped via linear filters (computed using the input shaping techniques), the obtained "shaped" joint motions are then used to compute a feedforward control torque (obtained via a classical "rigid" computed torque technique), finally, this torque law is added to a feedback joint friction compensator (based on a sliding mode controller). This 
"three stage" controller leads to good tracking of the desired joint trajectory, with the persistence of small tip oscillations. In [16], the point to point end-effector motion has been treated for the linear model of one-link arm. The authors searched for a proper end-effector trajectory cancelling the effect of the unstable zeros corresponding to the joint torque to end-effector transfer function, yielding stable inversion of this non-minimum phase dynamics. In [17], the authors proposed a closed-form solution for the point to point motion in fixed time problem when dealing with the linear model of a one-link robot. The scheme used is based on the parametrization of linear differential operators. We cite also the work [18], where the rest to rest motion in fixed time has been treated for a linear one-flexible link model. The idea is based on the design of an auxiliary output such that the associated transfer function has no zeros. The same approach has been extended in [19] to the particular nonlinear case of a two-link robot with a flexible forearm, when considering one elastic vibration mode to model the arm flexibility. The authors searched for a set of two outputs with respect to which the system has no zero dynamics, leading then to a closed-form solution for the rest to rest motion problem.

The above mentioned works are based either on linear models theories (i.e., most of the shaped inputs works), or on particular nonlinear models (i.e., multi-link rigid robots with a flexible forearm), and allow to minimize the residual tip vibrations in this case. However, no one permits to cancel these vibrations in a significant manner on a flexible multi-link testbed, using only joint measurements.

In this work is proposed a simple nonlinear model based solution to the rest to rest motion in fixed time problem for multi-link flexible planar manipulators. The idea is based on backward integration of the nonlinear elastic dynamics, starting from the desired final rest position. Then, the elastic states obtained are used to compute a feedforward control, which is added to simple joint state feedback (ensuring local exponential stability of the error dynamics equilibrium point). The whole controller is able to drive the system along the desired states, solving directly the rest to rest motion in fixed time problem when dealing with relatively slow joints motions. However, for faster joint positioning, the initial desired elastic states obtained through backward integration become large. Thus, the local controller fails to stabilize the system dynamics along the desired states. To overcome this limitation without introducing elastic state feedback (i.e., keeping the same simple locally stabilizing feedback), we propose to shape the joint trajectory, through an optimal planning technique, the goal being the minimization of the initial elastic states. The nice behavior of this approach is illustrated by the numerical simulation and experiments on a flexible two-link manipulator.

The paper is organized as follows. Section 2 recalls briefly the dynamics of planar multi-link flexible manipulators. In Sec. 3, the proposed scheme is presented. Section 4 is devoted to the numerical and experimental results obtained for the two-link case. Finally, conclusions are driven out in Sec. 5.

\section{Dynamic Modeling}

We recall in the following the general model form for planar arms, without going deeply into the model derivation. For details we refer the reader to [20] for small elastic displacement field models and [21] for large elastic displacements models. We consider a multi-body system composed of $n$ flexible links $S_{1}, \ldots, S_{n}$ loaded by a rigid body $S_{n+1}$. The base $S_{0}$ is supposed to be rigid as well as the $n$ joints. A reference frame $F_{i}, i$ $\in\{1, \ldots, n+1\}$ is rigidly attached to each body, to describe its position in the motion plant (Fig. 1). The elastic deformation in $S_{i}, i \in\{1, \ldots, n\}$ is represented as:

$$
\omega^{i}(x, t)=\phi^{i}(x) Q^{i}(t)
$$

with, $\phi^{i}$ the row vectors of shape functions (for detailed discussion about shape functions choice see $([20,22])$ and $Q^{i}$ the column

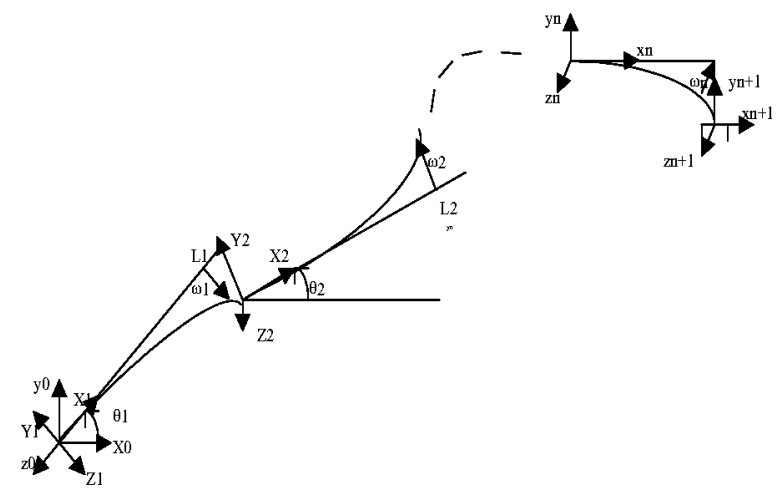

Fig. 1 Planar multi-link flexible manipulator

vector of elastic coordinates. Lagrangian formulation can be used to determine the final equations of motion, which are of the following general form:

$$
M(q) \ddot{q}+h_{c}(q, \dot{q})+K q=W u
$$

where, $q=\left(\theta_{1}, \ldots \theta_{n}, q_{11}, \ldots, q_{1 m_{1}}, \ldots, q_{n 1}, \ldots q_{n m_{n}}\right)^{T}$ is the vector of generalized coordinates, $u=\left(u_{1}, \ldots, u_{n}\right)^{T}$ the vector of joint torques, $M$ the positive definite symmetric inertia matrix, $h_{c}$ the vector of Coriolis and centrifugal forces, $K$ the stiffness matrix and $W$ the input weighting matrix which, due to the choice of clamped shape functions, assumes the form $\left[I_{n \times n}, 0_{n \times n_{e}}\right]^{T}$ (where $n_{e}=m_{1}+\ldots+m_{n}$ and $I$ is the identity matrix ${ }^{1}$ ).

Remark. We point out at this level that, contrary to some available methods (e.g., the shaped input technique), we do not need to take into account the elastic damping terms. This yields more interest to the obtained results, firstly because of the identification difficulties of these coefficients, leading to uncertain values [5], and secondly if the control scheme is able to damp out the residual oscillation without internal damping, things can not be worst with the presence of this damping effect in the simulation model and in the real system.

The mass matrix $M$, the stiffness matrix $K$ and the Coriolis and centrifugal vector $h_{c}$ can be partitioned consistently with the definition of $q$ as:

$$
\begin{gathered}
M=\left(\begin{array}{cc}
M_{r r} & M_{r e} \\
M_{r e}^{T} & M_{e e}
\end{array}\right) \quad K=\left(\begin{array}{cc}
0 & 0 \\
0 & K_{e e}
\end{array}\right) \\
h_{c}=\left(h_{r}, h_{e}\right)^{T}
\end{gathered}
$$

then, the dynamics can be rewritten separately for the rigid and flexible parts as follows:

$$
\begin{gathered}
M_{r r} \ddot{\Theta}+M_{r e} \ddot{Q}+h_{r}(\Theta, Q, \dot{\Theta}, \dot{Q})=I_{n \times n} \cdot u \\
M_{r e}^{T} \Theta+M_{e e} \ddot{Q}+h_{e}(\Theta, Q, \Theta, \dot{Q})+K_{e e} Q=0
\end{gathered}
$$

where $I_{n \times n}$ is the $n \times n$ identity matrix. and:

$$
\begin{gathered}
\Theta=\left(\theta_{1}, \ldots \theta_{n}\right)^{T} \\
Q=\left(q_{11}, \ldots, q_{1 m_{1}}, \ldots, q_{n 1}, \ldots q_{n m_{n}}\right)^{T}
\end{gathered}
$$

In the following section, we state the control problem as well as the solutions we are proposing both for slow and fast joint motions.

\footnotetext{
${ }^{1}$ In the following, $A_{n \times m}$ denotes a matrix with $n$ lines, $m$ columns.
} 


\section{The Control Scheme}

3.1 Rest to Rest Motion Problem Statement. Given a set of desired initial and final angular positions:

$$
\begin{aligned}
& \Theta_{d}\left(t_{0}\right)=\left(\theta_{d_{1}}\left(t_{0}\right) \ldots \theta_{d_{n}}\left(t_{0}\right)\right)^{T} \\
& \Theta_{d}\left(t_{f}\right)=\left(\theta_{d_{1}}\left(t_{f}\right) \ldots \theta_{d_{n}}\left(t_{f}\right)\right)^{T}
\end{aligned}
$$

where $t_{0}, t_{f}$ are respectively the fixed initial and final motion times, find a control law such that:

$$
\left\{\begin{array}{l}
\Theta(t)=\Theta_{d}\left(t_{0}\right) \text { for } t \leqslant t_{0}, \quad \Theta_{d}(t)=\Theta_{d}\left(t_{f}\right) \text { for } t \geqslant t_{f} \\
\Theta\left(t_{0}\right)=0_{n \times 1}, \quad \Theta\left(t_{f}\right)=0_{n \times 1} \\
Q(t)=0_{n_{e} \times 1} \text { for } t \leqslant t_{0}, \quad Q(t)=0_{n_{e} \times 1}, \quad \text { for } t \geqslant t_{f} \\
\dot{Q}\left(t_{0}\right)=0_{n_{e} \times 1}, \quad \dot{Q}\left(t_{f}\right)=0_{n_{e} \times 1}
\end{array}\right.
$$

Namely, we are searching for a causal control, implying a motion between the equilibrium points $\left(\Theta\left(t_{0}\right), Q\left(t_{0}\right)\right)=\left(\Theta_{d}\left(t_{0}\right), 0_{n_{e} \times 1}\right)$, $\left(\Theta\left(t_{f}\right), Q\left(t_{f}\right)\right)=\left(\Theta_{d}\left(t_{f}\right), 0_{n_{e} \times 1}\right)$, in a desired fixed time $t_{f}$.

3.2 Proposed Solution for Slow Motion. The control law used here is basically a feedforward plus feedback term based on the joint states. The main contribution of this work concerns the construction of the feedforward term. Considering a desired set of initial-final angular positions given by Eq. (6), this set can be interpolated through a fifth order polynomial trajectory: $\theta_{d_{i}}(t)$ $=\theta_{d_{i}}\left(t_{f}\right)\left(10\left(t / t_{f}\right)^{3}-15\left(t / t_{f}\right)^{4}+6\left(t / t_{f}\right)^{5}\right), i \in\{1 \ldots n\}$ satisfying the following classical velocity and acceleration constraints:

$$
\left\{\begin{array}{l}
\Theta_{d}^{(1)}\left(t_{0}\right)=\Theta_{d}^{(1)}\left(t_{f}\right)=0_{n \times 1} \\
\Theta_{d}^{(2)}\left(t_{0}\right)=\Theta_{d}^{(2)}\left(t_{f}\right)=0_{n \times 1}
\end{array}\right.
$$

Let:

$$
\Theta_{d}(t)=\left(\theta_{d_{1}}(t) \ldots \theta_{d_{n}}(t)\right)^{T}, \quad t \in\left[\begin{array}{ll}
t_{0} & t_{f}
\end{array}\right]
$$

represent the vector of the obtained joint trajectory. Then, using (9) the feedforward control can be directly obtained from Eq. (4), and writes:

$$
u_{f f}=M_{r r}^{d} \ddot{\Theta}_{d}+M_{r e}^{d} \dot{Q}_{d}+h_{r d}\left(\Theta_{d}, Q_{d}, \dot{\Theta}_{d}, \dot{Q}_{d}\right)
$$

where:

$$
\begin{aligned}
& M_{r r}^{d}=M_{r r}\left(\Theta_{d}, Q_{d}\right) \\
& M_{r e}^{d}=M_{r e}\left(\Theta_{d}, Q_{d}\right)
\end{aligned}
$$

and $Q_{d}$ represents the desired elastic vector associated to the desired joint trajectory and satisfies the following equation:

$$
\left(M_{r e}^{d}\right)^{T} \ddot{\Theta}_{d}+M_{e e}^{d} \ddot{Q}_{d}+h_{e d}\left(\Theta_{d}, Q_{d}, \Theta_{d}, \dot{Q}_{d}\right)+K_{e e} Q_{d}=0
$$

with,

$$
M_{e e}^{d}=M_{e e}\left(\Theta_{d}, Q_{d}\right)
$$

The idea we introduce here is the following: since the goal of control is joint motion between two equilibrium points set with elimination of the elastic vibration at the end of the desired motion, we propose to proceed through backward integration of the elastic dynamics Eq. (11), starting from the desired rest configuration, namely:

$$
Q_{d}\left(t_{f}\right)=0_{n_{e} \times 1}, \quad \dot{Q}_{d}\left(t_{f}\right)=0_{n_{e} \times 1}
$$

the corresponding final conditions differential equation system is then solved to obtain the desired elastic trajectory $Q_{d}(t)$. It is expected then that $Q_{d}(t), \dot{Q}_{d}(t)$ assume non zero initial conditions $Q_{d}\left(t_{0}\right), \dot{Q}_{d}\left(t_{0}\right)$, leading then to initial state error. However, the actual trajectory will be driven to reach the computed desired one, using an angular feedback regulator. The final control writes:

$$
u_{c l}(t)=u_{f f}(t)+K(t) e(t)
$$

where $e(t)=\left(\Theta_{d}(t)-\Theta(t), \dot{\Theta}_{d}(t)-\dot{\Theta}(t)\right)^{T}$ and $K(t)$ is a time dependent diagonal gain matrix, which is computed such as to ensure local stability of the linearized system along the desired state trajectory (this is possible due to the local controllability property of planar flexible arms [23]). When this holds and under sufficiently slow motion, local uniform exponential stability is ensured for the nonlinear system along the desired states ([24], pp. 242,246 and [25]).

Remarks. 1-Even if exponential convergence to the desired state trajectory is obtained for the system dynamics, it is clear that one would not be able to satisfy exactly the final time elastic constraints of system Eq. (7). However, the final elastic state values will be close to zero, leading then to negligible residual oscillations (see Sec. 4).

2-As we have explained before, this scheme does not need to take into account the damping terms, to reach the desired control goals. However, we should underline that when introducing linear elastic damping terms in the elastic dynamics Eq. (11), the backward integration could become critical. In fact, the linear damping terms yield asymptotic vanishing of the elastic trajectory, when integrated forward in time. Then, the backward integration will lead to unstable trajectory, if important damping terms are considered. However, when dealing with realistic damping coefficients values, their effect on the bounded integration domain $\left[t_{0}, t_{f}\right]$ remains small, yielding bounded feasible elastic states (refer to [26] for some numerical results). We insist then on the fact that these damping terms are absolutely not necessary to the control design we propose here so that we can make them vanish without worrying about.

The solution proposed above is appropriate when dealing with slow joint motions. The problem remains for fast motion. In fact, in this case system vibrations are expected to be more important and thus the elastic position and velocity $Q_{d}\left(t_{0}\right), \dot{Q}_{d}\left(t_{0}\right)$ to have large values, since the joint feedback ensures only local stability of the error dynamics equilibrium point, the actual system states could then drift off from the desired trajectory. To overcome this problem, keeping nevertheless the simple joint feedback, we propose to search for a proper angular trajectory such that it minimizes the initial elastic position and velocity $Q_{d}\left(t_{0}\right), \dot{Q}_{d}\left(t_{0}\right)$. In the following we present how to generate these optimal joint trajectories.

\subsection{Optimal Joint Trajectory Generation for Fast Rest-to- Rest Motion}

3.3.1 Formulation as Pontryagin Optimal Problem. We are searching for a joint trajectory satisfying the constraints Eq. (6), Eq. (8) and minimizing the cost $J$ :

$$
J=\frac{1}{2}\left(Q_{d}\left(t_{0}\right)^{T} \cdot K_{1} \cdot Q_{d}\left(t_{0}\right)+\dot{Q}_{d}\left(t_{0}\right)^{T} \cdot K_{2} \cdot \dot{Q}_{d}\left(t_{0}\right)\right)
$$

where $K_{1}, K_{2}$ are two positive definite matrices.

To solve this problem we define a general polynomial form for the joint trajectory:

$$
\begin{aligned}
\theta_{d_{k}}(t)= & \sum_{i=0}^{i=6} a_{i} \cdot\left(t / t_{f}\right)^{i}+\sum_{j=1}^{j=m} b_{j} \cdot\left(t / t_{f}\right)^{(j+6)}, \\
& k \in\{1 \ldots n\}, \quad m \in \mathbb{N}^{*}
\end{aligned}
$$

The $\theta_{d k}(t)$ can be expressed as functions of the $\left(b_{i}\right)$ coefficients by substituting Eq. (15) into Eq. (6), Eq. (8) and solving for the $\left(a_{i}\right)$ vector. Using the obtained joint trajectory $\theta_{d i}(t, B)$ (where $\left.B=\left(b_{1} \ldots b_{m}\right)\right)$, together with Eq. (11) we can formulate the optimization problem presented above as the following Pontryagin optimal control problem ([27], p. 315): 


$$
\begin{gathered}
\min _{B}\left(J=\frac{1}{2}\left(X_{1}\left(t_{0}\right)^{T} \cdot K_{1} \cdot X_{1}\left(t_{0}\right)+X_{2}\left(t_{0}\right)^{T} \cdot K_{2} \cdot X_{2}\left(t_{0}\right)\right)\right) \\
\dot{X}=F(t, X, B), \quad X\left(t_{f}\right)=0_{n_{e} \times 1}, \quad B \in R^{m},
\end{gathered}
$$

$$
(t, X) \in\left[t_{0}, t_{f}\right] \times R^{2 . n_{e}}
$$

where

$$
F(t, X, B)=\left(\begin{array}{c}
X_{2} \\
\left(M_{e e}^{d}\right)^{-1}\left(t, X_{1}, B\right)\left(-M_{r e}^{d}\left(t, X_{1}, B\right)^{T} \ddot{\Theta}_{d}(t, B)-h_{e d}(t, X, B)-K_{e e} X_{1}\right)
\end{array}\right)
$$

and $B$ is considered as a constant control vector over $\left[t_{0}, t_{f}\right]$, to be determined such that minimizing the cost $J$. We refer to [26], for a solution existence analysis.

When the optimal trajectory has been obtained, one can proceed through backward integration as explained in Sec. 3.2. Since the trajectory has been designed such as to minimize the initial elastic states, a simple joint feedback will be enough to drive the system from the desired point to the goal through the optimal trajectory.

In the following section we present numerical as well as experimental results obtained for a two-link flexible arm.

\section{Simulation and Experimental Results}

4.1 Testbed Description. The arm (see Fig. 2) is characterized by mechanical properties summarized in Table 1. It is driven by D.C. servo motors. For real time law computation a PC 486 is used, with 12 bit A/D and D/A boards. The sampling period used is $5 \mathrm{~ms}$. The angular positions are measured by encoder of 14400 points and 2000 points for the first and second joint respectively. The angular velocity is computed through basic numerical differentiation and filtering. Strain gauges are used to measure the tip elastic displacements.

4.2 Simulation Results. The dynamical model of the arm described above has been computed using two clamped modes for each arm. First, we start by testing the proposed approach for

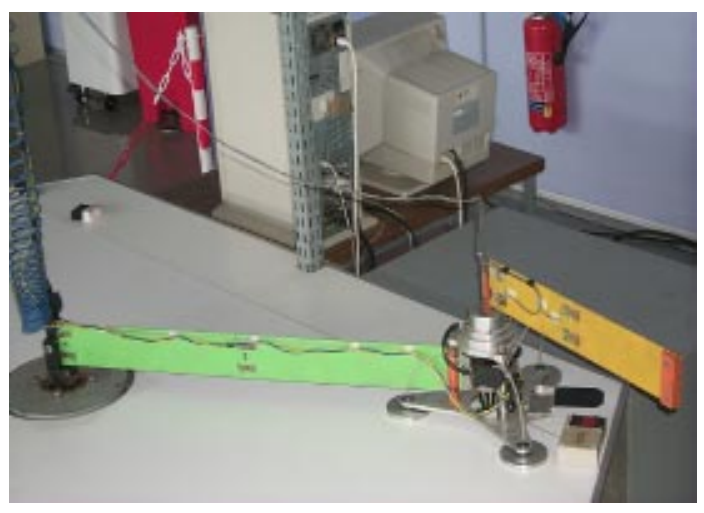

Fig. 2 The two-link flexible arm testbed

Table 1 Mechanical properties of the arm

\begin{tabular}{lll}
\hline \hline Property & First Link & Second Link \\
\hline Length. L $(\mathrm{m})$ & 1.005 & 0.52 \\
Mass density. $\rho(\mathrm{kg} / \mathrm{m})$ & 2.032 & 0.706 \\
Rigidity. EI $\left(\mathrm{N} \cdot \mathrm{m}^{2}\right)$ & 47.25 & 1.749 \\
Tip-load mass. $m_{\text {tip }}(\mathrm{kg})$ & 7.66 & 0.51 \\
Tip-load inertia. $J_{\text {tip }}\left(\mathrm{kg} \cdot \mathrm{m}^{2}\right)$ & $171 \mathrm{e}-3$ & $623 \mathrm{e}-7$ \\
Hub inertia. $J_{h}\left(\mathrm{~kg} . \mathrm{m}^{2}\right)$ & $1.8 \mathrm{e}-3$ & $225 \mathrm{e}-6$ \\
Arm thickness. $d(\mathrm{~mm})$ & 3 & 1 \\
\hline \hline
\end{tabular}

relatively slow motion. We have chosen as desired angular equilibrium points $\Theta_{0}=(0)_{(2,1)} \mathrm{rad}, \Theta_{f}=(\pi / 2, \pi / 2)^{T} \mathrm{rad}$.

Let us recall that by relatively slow, we mean motions slow enough in order that the initial elastic states, that will hold after the backward integration, remain small. To determine, at least numerically, a threshold for such a value of the time motion, we have computed the square of the elastic vector $\left(Q_{d}\left(t_{0}\right), \dot{Q}\left(t_{0}\right)\right)$ norm, as a function of the final motion time $t_{f}$. The obtained result is displayed in Fig. 3. One can see that the value $t_{f}=3 \mathrm{~s}$ seems to be a critical one. To begin with, we test a slow trajectory (Fig. 4), fixing $t_{f}=5 \mathrm{~s}$. We pursue by testing a faster motion corresponding to the critical value $t_{f}=3 \mathrm{~s}$.

The procedure described in Sec. 3.2 has been run to compute the elastic trajectory associated to the joint desired motion. Figures 5 and 6 display the joint trajectory tracking obtained for a nominal simulation plant, without introducing elastic damping coefficients in the simulation model. It is clear that the angular final position is reached with small error. The tip elastic displacements are displayed in Figs. 7 and 8. One can see that these deflections

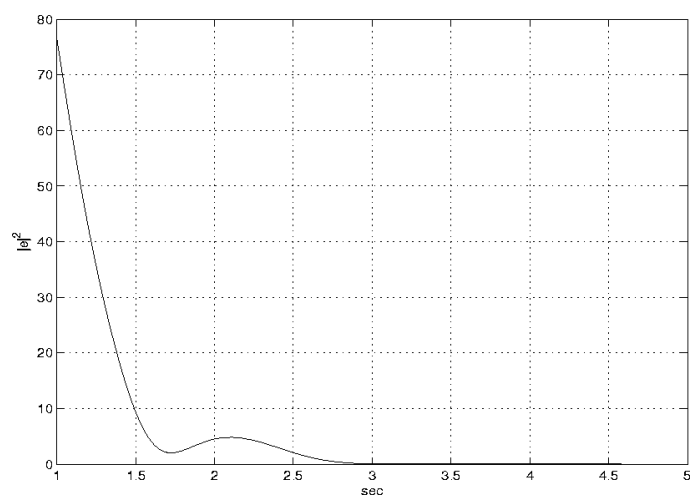

Fig. 3 Initial elastic vector Euclidian norm square $|e|^{2}$ as function of $t_{f}$

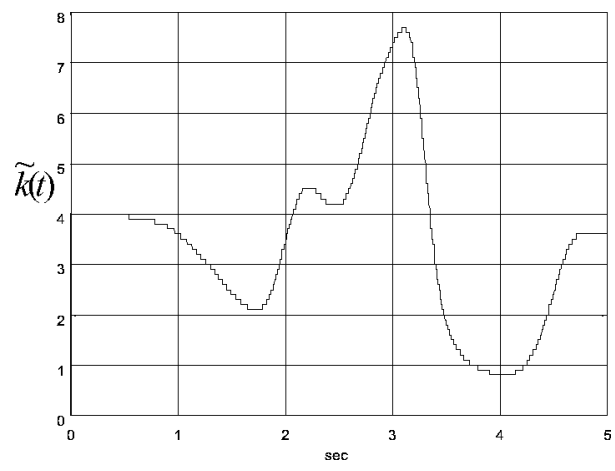

Fig. 4 Time scheduling gain, slow motion $t_{f}=5 \mathrm{~s}$ 


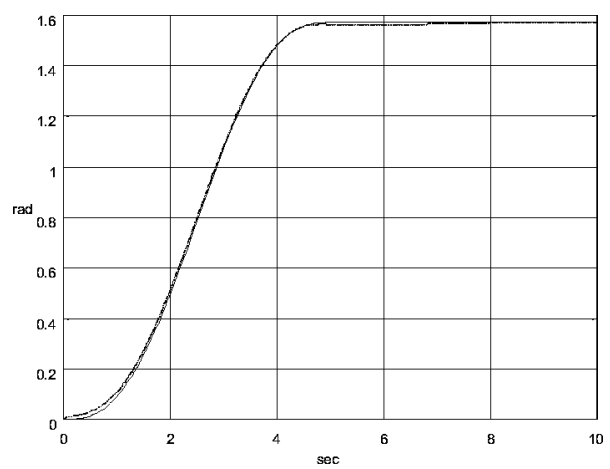

Fig. 5 First joint motion, desired (continuous line) and actual (dashed line) motion, slow motion $t_{f}=5 \mathrm{~s}$, simulation results

are damped out at the end of the desired rigid motion. Even if, as discussed in Sec. 3.2, the tip oscillations do not exactly vanish at the final instant, these residual amplitudes are quite negligible. The corresponding smooth closed-loop torques are given in Figs. 9 and 10. These torques do not vanish at the initial instant: this is due to the nonzero elastic positions and velocities initial values obtained through the backward integration, yielding via Eq. (10) to a nonzero initial feedforward. Figure 4 displays the obtained time varying gain $(\widetilde{k}(t)$, s.t., $K$ in Eq. (13) writes $K=\widetilde{k}(t)$ $\left.\times\left[I_{n \times n}, 0.5 I_{n \times n}\right]\right)$, computed such that at each time sample, the linearized error dynamics be stable (see discussion in Sec. 3.2). It is clear that the local stability does not require high gain values, which is quite important for practical implementation. We have also done some simulations including perturbations with respect to model parameters, initial values uncertainties and measurement noises, by introducing the following disturbances:

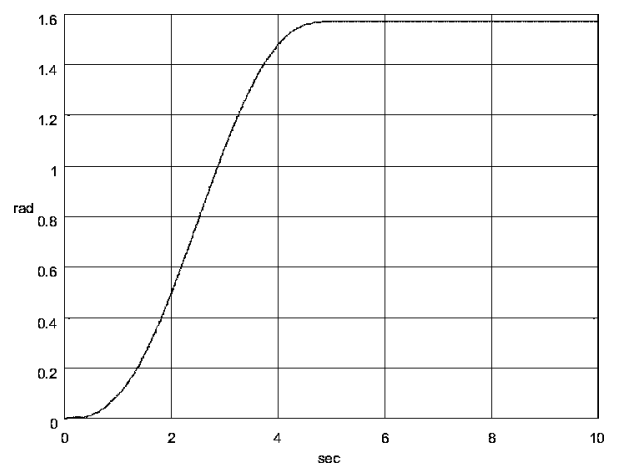

Fig. 6 Second joint motion, desired (continuous line) and actual (dashed line) motion, slow motion $t_{f}=5 \mathrm{~s}$, simulation results

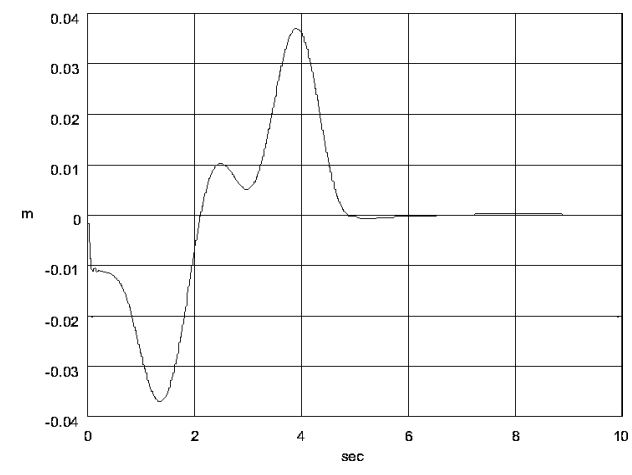

Fig. 7 First arm tip-deflection, slow motion $t_{f}=5 \mathrm{~s}$, simulation results

Journal of Dynamic Systems, Measurement, and Control

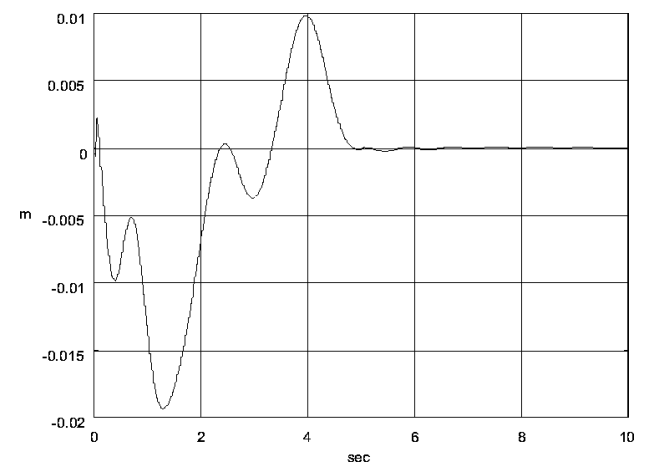

Fig. 8 Second arm tip-deflection, slow motion $t_{f}=5 \mathrm{~s}$, simulation results

- $\delta m_{\text {tip } 1}=\delta m_{\text {tip } 2}=+10 \%$ (more loaded arms)

- $\delta E I_{1}=\delta E I_{2}=-20 \%$ (more flexible rigid arms)

- $1.05 \mathrm{~cm}$ and $0.6 \mathrm{~cm}$ of initial tip-displacements for the first and second link respectively (corresponding to $\delta \theta_{1}\left(t_{0}\right)=\delta \theta_{2}\left(t_{0}\right)$ $=10^{-2} \mathrm{rad}$ and, $\delta q_{11}\left(t_{0}\right)=\delta q_{21}\left(t_{0}\right)=10^{-3}, \delta q_{12}\left(t_{0}\right)=\delta q_{22}\left(t_{0}\right)$ $=0$ ).

- Angular position and velocity measurements noises, modelled by a random variable uniformly distributed between $\pm 1.610^{-5}$. The desired joint positioning constraints are still satisfied as displayed in Figs. 11 and 12. However, tip vibrations appear for the second arm (see Figs. 13 and 14), but remain small (keeping in mind that no elastic damping has been introduced). The feasible closed-loop controls are given in Figs. 15 and 16. The collocated feedback seems to give robustness to the whole controller with respect to the considered uncertainties. However, for larger disturbances one should think about an adaptive version of this scheme, e.g. [28].

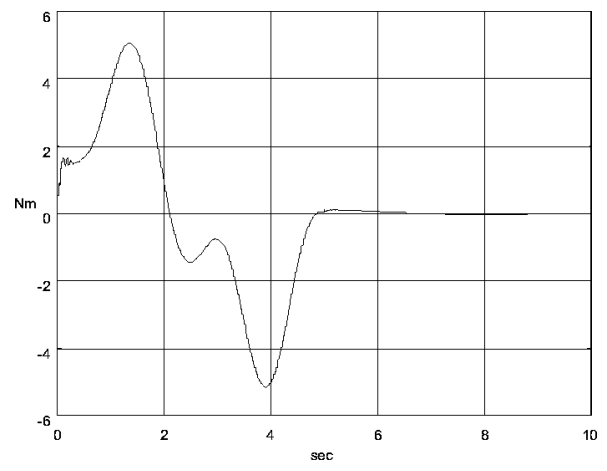

Fig. 9 First closed-loop torque, slow motion $t_{f}=5 \mathrm{~s}$, simulation results

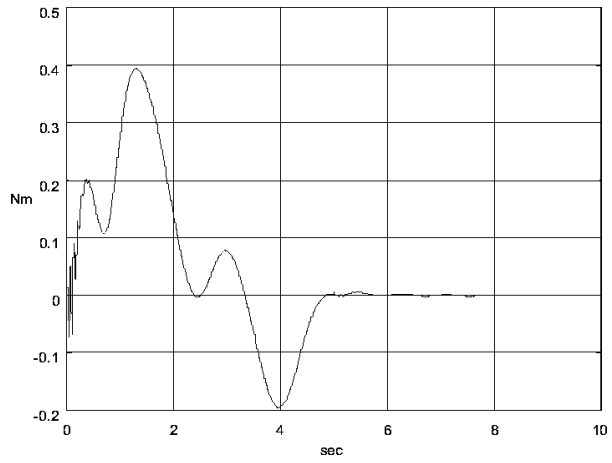

Fig. 10 Second closed-loop torque, slow motion $t_{f}=5 \mathrm{~s}$, simulation results 


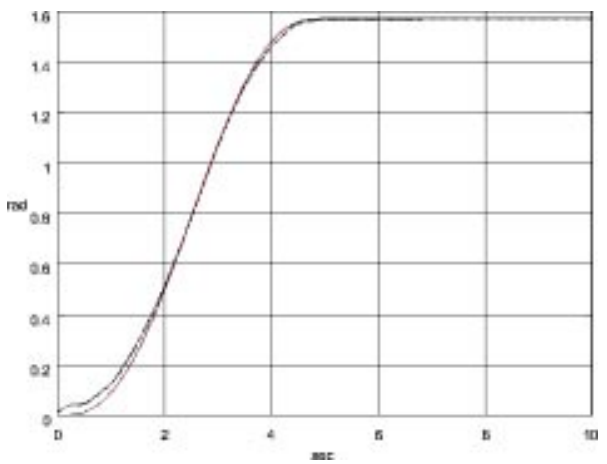

Fig. 11 First joint motion, desired (continuous line) and actual (dashed line) motion, disturbed plant

Consider now a faster motion case, namely the travelling time $t_{f}=3 \mathrm{~s}$. To display the necessity of the optimal trajectory shaping, introduced in Sec. 3.3, we first report the obtained results associated to a direct application of the backward integration approach, with the fifth order polynomial joint trajectory (i.e., without trajectory planning). Figure 17 shows the first angular motion. As expected (see Sec. 3) the joint constraints of the rest to rest problem Eq. (7) are not achieved with good precision. We turn then to the application of the optimal planning scheme presented in Sec. 3.3 and search for an optimal joint vector trajectory Eq. (15) solving the problem defined by Eq. (16). To display the effect of the vector $B$ dimension on the optimal trajectory, we have compared the trajectories obtained for different values of index $m$. It is clear from Fig. 18 that changing the number of optimization parameter, does not affect significantly the obtained optimal trajectory. We have kept then the dimension $m=5$ (which seems to yield a lower optimization time). The optimal problem has been solved using the Nelder-Mead simplex method, a nonlinear unconstrained

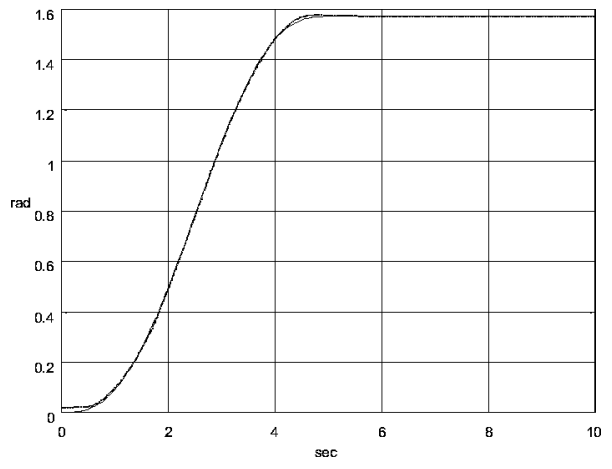

Fig. 12 Second joint motion, desired (continuous line) and actual (dashed line) motion, disturbed plant

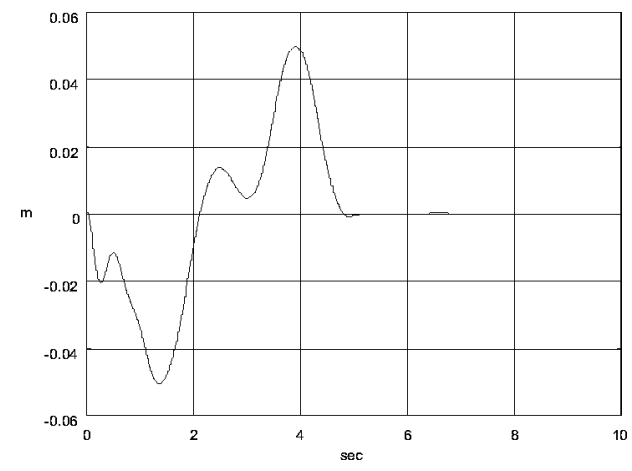

Fig. 13 First link tip deflection, disturbed plant

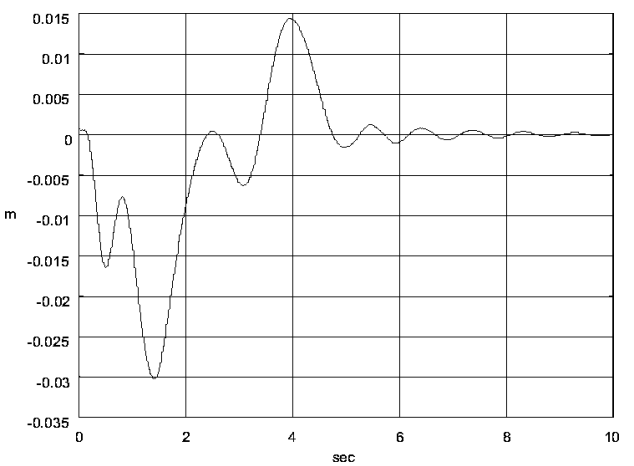

Fig. 14 Second link tip deflection, disturbed plant

minimization code, based on a direct search method (see for eg. [29]). The obtained simulation results on the nominal plant are given in Figs. 19 to 24. Figures 19 and 20 display the obtained joint motions. In Figs. 21 and 22, we can see that the elastic displacement of the end-effector vanishes at the end of the motion. The corresponding closed-loop control torques and varying gain are given in Figs. 23, 24 and 25. The maximum torque values are clearly (and logically) larger than those obtained for the first motion. This can be enhanced by choosing a less demanding joint trajectory or with a slight modification of the optimization cost. In fact, following e.g. $[30,14]$, one can replace the polynomial joint trajectory Eq. (15) with a near bang-bang function (i.e., bang-bang acceleration with smooth commutation functions), this allows to realize the same joint motions with lower requested torques, this being due to the uniform acceleration distribution (see [30] for more details). Another classical solution could proceed through the modification of the optimization cost Eq. (14) as follows [31]: $\widetilde{J}=J+1 / t_{f} \int_{0}^{t_{f}} \sum_{i=1}^{i=n}\left(u_{i}(t) / u_{i \max }\right)^{2} d t$, where $J$ is given by Eq. (14), and $u_{i \max }, i \in\{1 . . n\}$ are the maximal acceptable torque values.

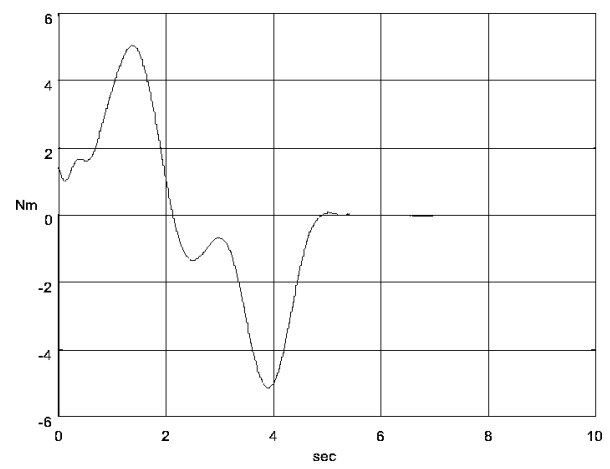

Fig. 15 First closed-loop torque, disturbed plant

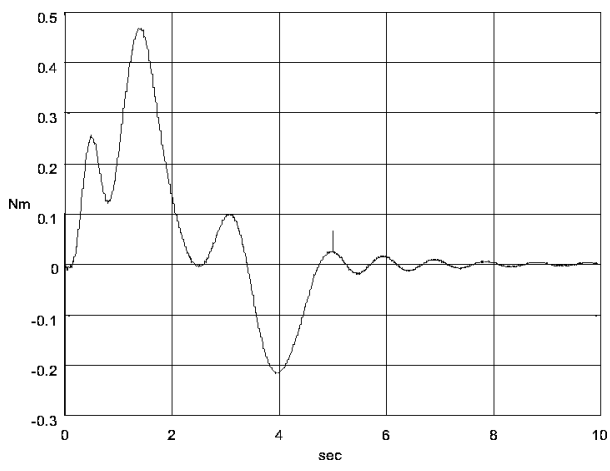

Fig. 16 Second closed-loop torque, disturbed plant 


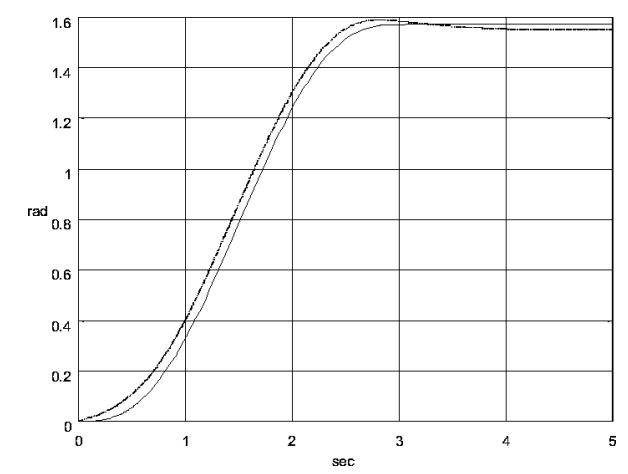

Fig. 17 Desired and actual (dashed line) motion, fast motion $t_{f}=3 \mathrm{~s}$ without optimal trajectory planning

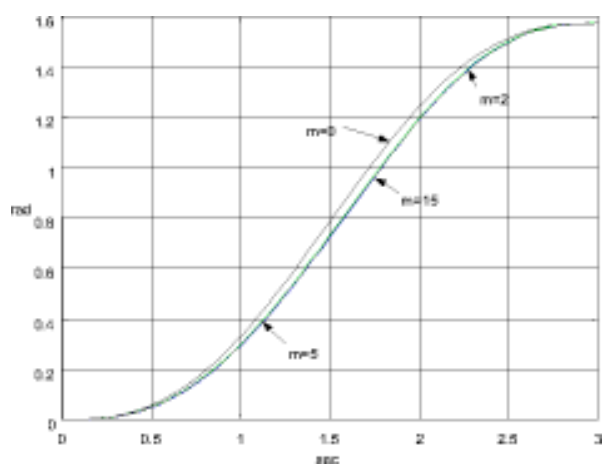

Fig. 18 Optimal trajectories for different $m$ values

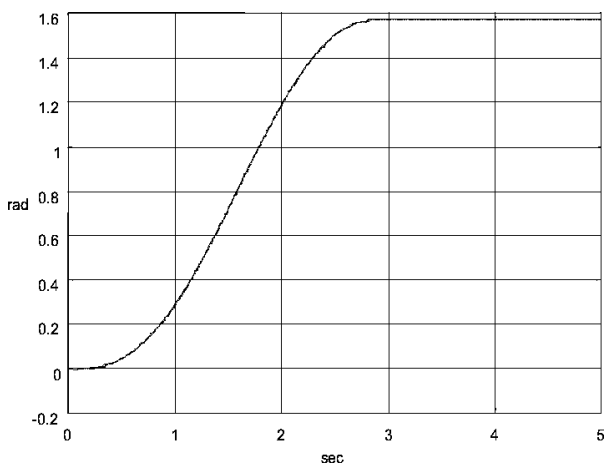

Fig. 19 First joint motion, desired (continuous line) and actual (dashed line) motion, fast motion $t_{f}=3 \mathrm{~s}$, simulation results

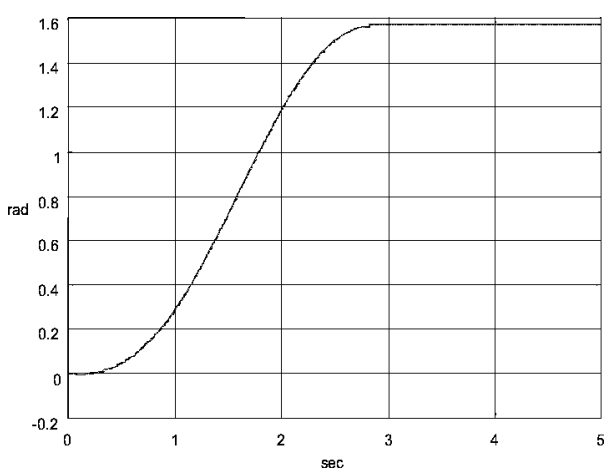

Fig. 20 Second joint motion, desired (continuous line) and actual (dashed line) motion, fast motion $t_{f}=3 \mathrm{~s}$, simulation results

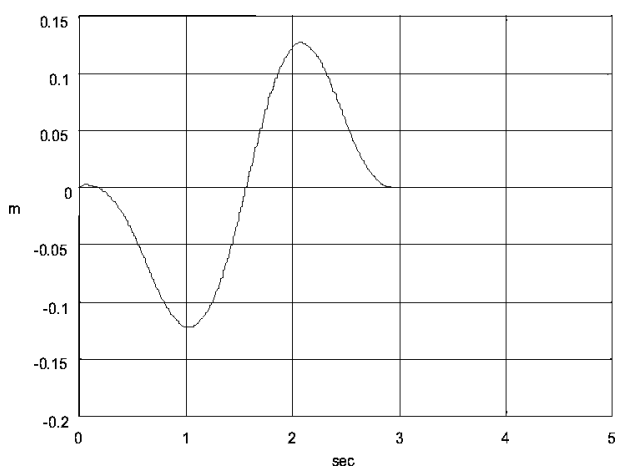

Fig. 21 First link tip deflection, fast motion $t_{f}=3 \mathrm{~s}$, simulation results

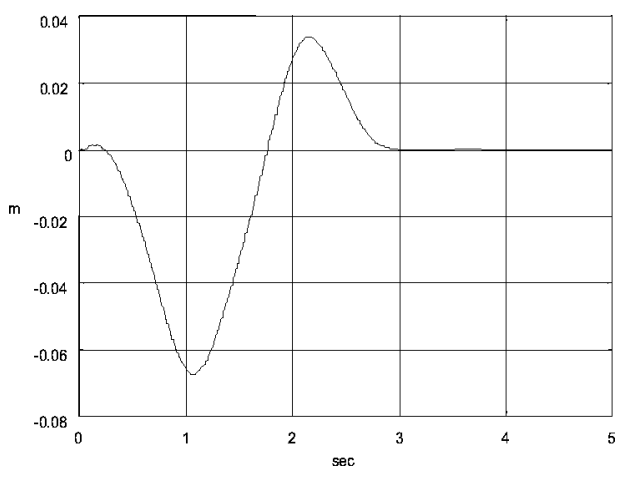

Fig. 22 Second link tip deflection, fast motion $t_{f}=3 \mathrm{~s}$, simulation results

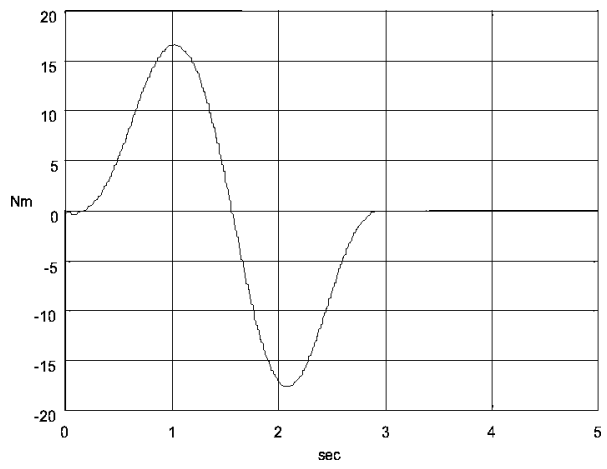

Fig. 23 First closed-loop torque, fast motion $t_{f}=3 \mathrm{~s}$, simulation results

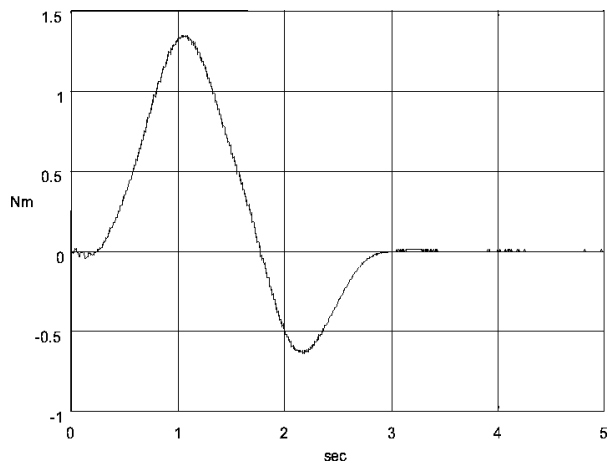

Fig. 24 Second closed-loop torque, fast motion $t_{f}=3 \mathrm{~s}$, simulation results 


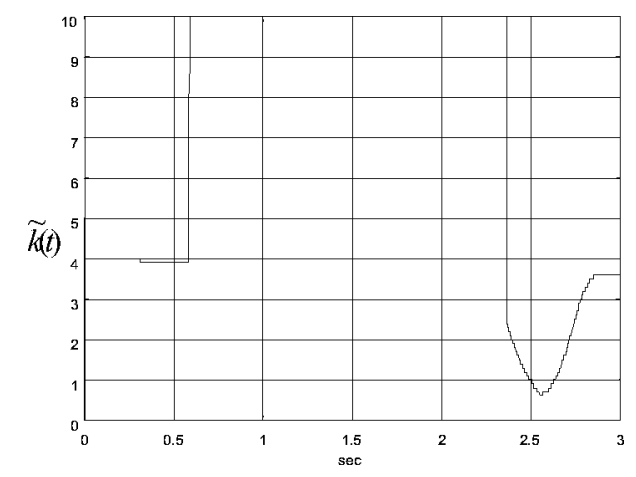

Fig. 25 Time scheduling gain, fast motion $t_{f}=3 \mathrm{~s}$

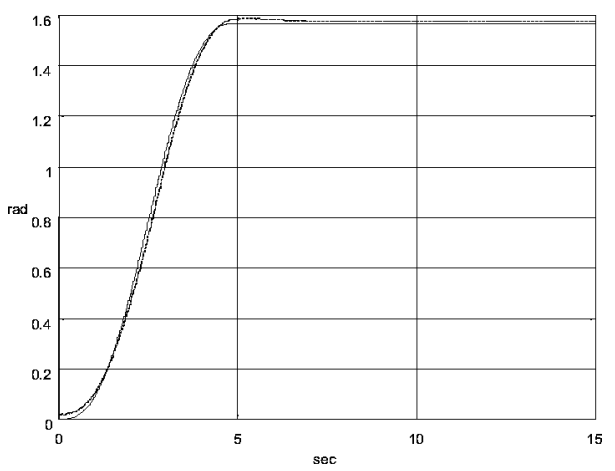

Fig. 26 First joint motion, desired (continuous line) and actual (dashed line) motion, slow motion $t_{f}=5 \mathrm{~s}$, experimental test

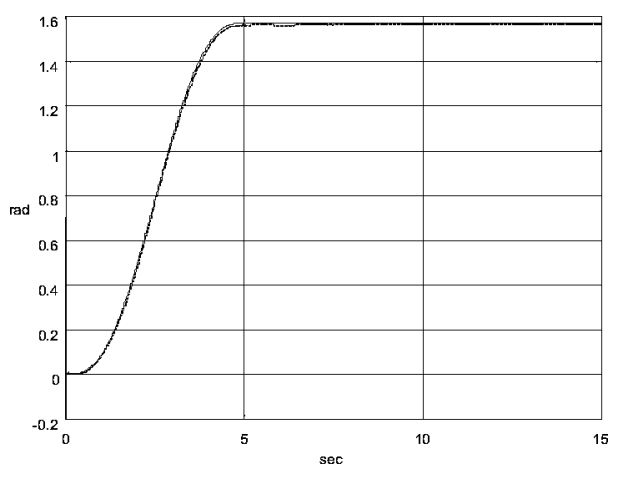

Fig. 27 Second joint motion, desired (continuous line) and actual (dashed line) motion, slow motion $t_{f}=5 \mathrm{~s}$, experimental test

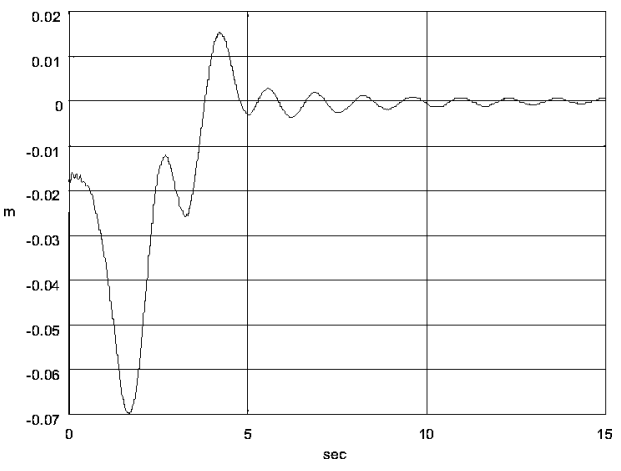

Fig. 28 First arm tip-deflection, slow motion $t_{f}=5 \mathrm{~s}$, experimental test

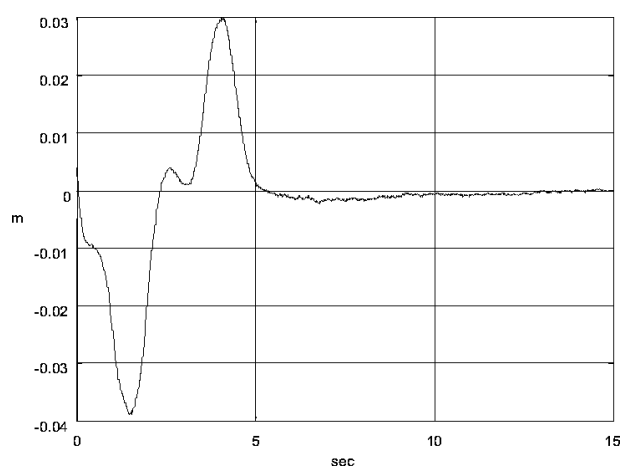

Fig. 29 Second arm tip-deflection, slow motion $t_{f}=5 \mathrm{~s}$, experimental test

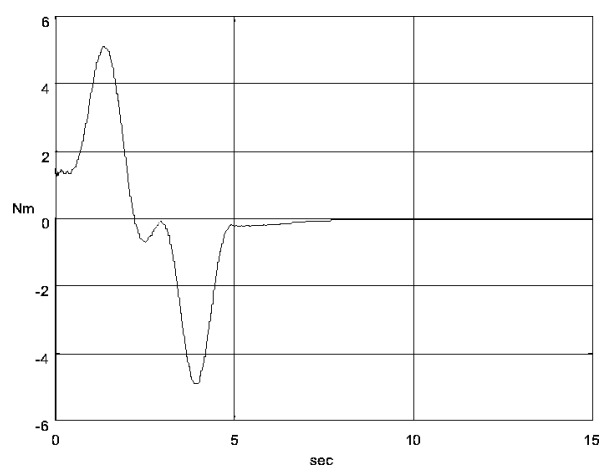

Fig. 30 First closed-loop torque, slow motion $t_{f}=5 \mathrm{~s}$, experimental test

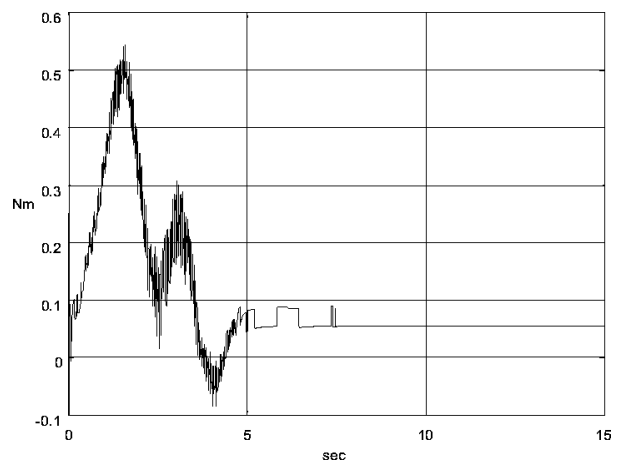

Fig. 31 Second closed-loop torque, slow motion $t_{f}=5 \mathrm{~s}$, experimental test

Through the minimization of $\widetilde{J}$ one can minimize the initial elastic coordinates as well as the torque values along the optimal joint trajectory.

4.3 Experimental Results. In this section are presented the experimental results. The rest to rest motion tested is characterized by a fifth order polynomial joint trajectory between the two equilibrium $\Theta_{0}=(0)_{(2,1)} \mathrm{rad}, \Theta_{f}=(\pi / 2, \pi / 2)^{T} \mathrm{rad}$ with a time motion of $5 \mathrm{~s}$ (i.e., the first simulation test). Due to PC memory constraints, we have replaced the varying gain with the constant joint gain feedback: $K_{\theta_{1}}=13 \mathrm{~N} . \mathrm{m} / \mathrm{rad}, K_{\dot{\theta}_{1}}=0.2 \mathrm{~N} . \mathrm{m} . \mathrm{s} / \mathrm{rad}, K_{\theta_{2}}$ $=10 \mathrm{~N} . \mathrm{m} / \mathrm{rad}, K_{\dot{\theta}_{2}}=0.2 \mathrm{~N} . \mathrm{m} . \mathrm{s} / \mathrm{rad}$. The good tracking performances obtained are displayed in Figs. 26 and 27. Furthermore, the elastic tip vibrations have been effectively damped out at the end of the desired motion (Figs. 28 and 29). The causal closedloop torques are presented in Figs. 30 and 31 and show a smooth 
behavior, with non zero initial values, due to the feedforward part (as explained in the previous section). Also, contrary to the simulation results, the practical torques present a small static value, due to the unmodeled joint frictions.

\section{Conclusion}

In this work is introduced a simple idea to solve the problem of rest to rest motion in fixed desired time for multi-link planar flexible manipulators. This problem amounts to realize joint motion between two equilibrium points in fixed desired time together with cancellation of the tips oscillations at the end of the desired joint motions. The solution proposed here achieves these two objectives and this, without any internal elastic damping. This scheme is based on backward integration of the elastic dynamics along the joint desired trajectory, starting from the desired rest position. Furthermore, the unique required measurements (for feedback purposes) are the joint states. The proposed scheme permits to directly achieve these goals, when dealing with slow motions. However, for fast joint trajectories, the direct application of the method did not show to be efficient. We have then proposed an optimal trajectory planning to overcome this limitation. The optimization planning statement has been formulated as a classical Pontryagin optimal control problem, yielding direct optimal solution existence. Numerical and experimental tests on a flexible two-link manipulator have shown the good behavior of the proposed ideas.

\section{References}

[1] Meckl, P., 1994. "Robust motion control of flexible systems using feedforward forcing functions.” IEEE Trans. Control Syst. Technol., 2(3), pp. 245-253.

[2] Chan, T., and Stelson, K., 1995. "Point to point motion commands that eliminate residual vibration." In American Control Conference, pp. 909-913.

[3] O'Connor, W., and Lang, D., 1998. "Position control of flexible robot arms using mechanical waves." ASME J. Dyn. Syst., Meas., Control, 120, pp. $334-$ 339 .

[4] Singer, N., and Seering, W., 1990. "Preshaping command inputs to reduce system vibration.” ASME J. Dyn. Syst., Meas., Control, 112, pp. 76-82.

[5] Pao, L., and Lau, M., 2000. "Robust input shaper control design for parameter variations in flexible structures." ASME J. Dyn. Syst., Meas., Control, 122, pp. 63-70.

[6] Zuo, K., Drapeau, V., and Wang, D., 1995. “Closed loop shaped input strategies for flexible robots." Int. J. Robot. Res., 14(05) October, pp. 510-529.

[7] Wang, D., and Vidyasagar, M., 1991. "Control of a class of manipulators with last link flexible-part I: Feedback linearization.” ASME J. Dyn. Syst., Meas., Control, 113, pp. 655-661.

[8] Wang, D., and Vidyasagar, M., 1991. "Control of a class of manipulators with last link flexible-part II: Observer-controller stability.” ASME J. Dyn. Syst., Meas., Control, 113, pp. 662-668.

[9] Chalhoub, N., and Ulsoy, A., 1987. "Control of a flexible robot arm: experimental and theoretical results." ASME J. Dyn. Syst., Meas., Control, 109 September, pp. 299-310.

[10] Schoenwald, D., Feddema, J., Eisler, G., and Segalman, D., 1991. “Minimum- time trajectory control of a two-link flexible robotic manipulator." In IEEE, Int. Conference on Robotics and Automation, pp. 2114-2120. Sacramento, California.

[11] Khorrami, F., 1990. "A two-stage controller for vibration suppression of flexible-link manipulators." In 29th IEEE, Conference on Decision and Control, pp. 2560-2565.

[12] Mohri, A., Sarkar, P., and Yamamoto, M., 1998. "An efficient motion planning of flexible manipulatore along specified path." In IEEE, Int. Conference on Robotics and Automation, pp. 1104-1109.

[13] Morris, A., and Madani, A., 1998. "Quadratic optimal control of a twoflexible-link robot manipulator.” Robotica, 16, pp. 97-108.

[14] Aoustin, Y., and Formalsky, A., 1999. "On the feedforward torques and reference trajectory for flexible two-link arm.” Multibody Syst. Dyn., 3 February, pp. $241-265$.

[15] Romano, M., Agrawal, B., and Bernelli-Zazzera, F., 2002. "Experiments on command shaping control of a manipulator with flexible links." J. Guid. Control Dyn., 25(2), pp. 232-239.

[16] Benosman, M., and Le Vey, G., 2003. "Stable inversion of SISO nonminimum phase linear dynamic through output planning: an experimental application to the one-link flexible manipulator." IEEE Trans. Control Syst. Technol., 11(4) pp. 588-597.

[17] Benosman, M., and Le Vey, G., 2000. "Exact articular trajectory tracking for a one-link flexible manipulator: an approach through the parametrization of differential operators." In 31st International Symposium on Robotics, pp. 493 498.

[18] De Luca, A., and Di Giovanni, G., 2001. "Rest to rest motion of a one-link flexible arm." In IEEE/ASME International Conference on Advanced Intelligent Mechatronics, pp. 923-928.

[19] De Luca, A., and Di Giovanni, G., 2001. "Rest-to-rest motion of a two-link flexible robot with a flexible forearm." In IEEE/ASME International Conference on Advanced Intelligent Mechatronics, pp. 929-935.

[20] De Luca, A., and Siciliano, B., 1991. "Closed-form dynamic model of planar multilink lightweight robots." IEEE Trans. Syst. Man Cybern., 21(4) July/ August, pp. 826-839.

[21] Boyer, F., and Glandais, N., 1999. "Simulation of flexible manipulators with elastic non-linearities." In IEEE, Int. Conference on Robotics and Automation.

[22] Bellezza, F., Lanari, L., and Ulivi, G., 1990. "Exact modeling of the flexible slewing link." In IEEE, Int. Conference on Robotics and Automation, pp. $734-739$.

[23] Lopez-Linares, S., Konno, A., and Uchiyama, M., 1994. "Controllability of flexible manipulators." In IFAC, Symposium on Robot Control, pp. 509-516.

[24] Khalil, H., 1996. Nonlinear systems, second ed. New York Macmillan.

[25] Paden, B., Chen, D., Ledesma, R., and Bayo, E., 1993. "Exponentially stable tracking control for multijoint flexible-link manipulators." ASME J. Dyn. Syst., Meas., Control, 115, pp. 53-59.

[26] Benosman, M., 2002. Control of flexible manipulators with extension to nonminimum phase systems. Tech. Rep. 02/1/AUTO, Ecole des Mines de Nantes. Thesis preprint.

[27] Alexeev, V., Tikhomirov, V., and Fomine, S., 1982. Commande Optimale, MIR ed.

[28] Damaren, C., 1996. "Adaptive control of flexible manipulators carrying large uncertain payloads." Int. Journal of Robotic Systems, 13(4), pp. 219-228.

[29] Lagarias, J., Reeds, J., Wright, M., and Wright, P., 1998. "Convergence properties of the nelder-mead simplex method in low dimensions." SIAM J. Optim., 09(01), pp. 112-147.

[30] Serna, M., and Bayo, E., 1989. "Trajectory shaping for flexible manipulators: a comparative study." In Fourth IEEE, Int. Conference on Robotics and Automation, pp. 159-174.

[31] Kinceler, R., and Meckl, P., 1995. "Input shaping for nonlinear systems." In American Control Conference, pp. 914-918. 